\title{
EAGLES OF \\ THE RAF \\ THE WORLD WAR II \\ EAGLE SQUADRONS
}

by

Philip D. Caine

\section{ndu \\ DRESS}

1991

National Defense University Press

Washington, DC 